\title{
Previously Unknown Ultra-low Energy Level of the Hydrogen Atom Whose Existence can be Predicted
}

\author{
Koshun Suto ${ }^{1}$ \\ ${ }^{1}$ Chudaiji Buddhist Temple, Isesaki, Japan \\ Correspondence: Koshun Suto, Chudaiji Buddhist Temple, 5-24, Oote-Town, Isesaki, 372-0048, Japan. \\ Tel: 81-270-23-9980. E-mail: koshun_suto129@mbr.nifty.com
}

Received: September 26, 2014 Accepted: November 5, $2014 \quad$ Online Published: November 11, 2014

doi:10.5539/apr.v6n6p64

URL: http://dx.doi.org/10.5539/apr.v6n6p64

\begin{abstract}
This paper theoretically predicts that if the energy level of the hydrogen atom is expressed classically as $E_{n}=-\alpha^{2} m_{\mathrm{e}} c^{2} / 2 n^{2}$, then the energy level $E_{n}=-2 m_{\mathrm{e}} c^{2}+\alpha^{2} m_{\mathrm{e}} c^{2} / 2 n^{2}$ exists in the hydrogen atom. Triplet production is an experiment which strongly supports the existence of an electron at this extremely low energy level. However, an interpretation different from the conventional interpretation is needed in order to regard triplet production as evidence for the prediction in this paper. If an electron can exist at an unknown energy level, then it can be predicted that hydrogen atoms in this state will be a strong candidate for dark matter.
\end{abstract}

Keywords: Einstein's energy-momentum relationship, Dirac relativistic wave equation, hydrogen atom, energy level, triplet production, dark matter

\section{Introduction}

Letting $m c^{2}$ be the relativistic energy and $\boldsymbol{p}$ the momentum of an object or a particle existing in free space, Einstein's energy-momentum relationship is given by the following equation:

$$
\left(m c^{2}\right)^{2}=\boldsymbol{p}^{2} c^{2}+\left(m_{0} c^{2}\right)^{2} \text {. }
$$

Here, $m_{0} c^{2}$ is the rest mass energy of an object or a particle.

In contrast, the author has derived the following relationship for the bound electron in a hydrogen atom, which must take into account the Coulomb potential (Suto, 2011):

$$
E_{\mathrm{re}, n}^{2}+\boldsymbol{p}_{n}^{2} c^{2}=\left(m_{\mathrm{e}} c^{2}\right)^{2}, \quad E_{\mathrm{re}, n}=m_{\mathrm{e}} c^{2}+E_{n}, \quad n=1,2, \cdots .
$$

Here, $E_{\mathrm{re}, n}$ is the relativistic energy of the electron, and the electron's energy is described on an absolute scale. In this case, it is natural to regard $\boldsymbol{p}$ as angular momentum.

In Equation (2), $E_{n}$ is the total mechanical energy of a hydrogen atom derived from classical quantum theory, and it is given by the following equation:

$$
E_{n}=-\frac{1}{2}\left(\frac{1}{4 \pi \varepsilon_{0}}\right)^{2} \frac{m_{\mathrm{e}} e^{4}}{\hbar^{2}} \frac{1}{n^{2}}, \quad n=1,2, \cdots .
$$

The relativistic energy of the hydrogen atom obtained from the Dirac relativistic wave equation is given by the following equation (Schiff, 1968):

$$
E=m_{\mathrm{e}} c^{2}\left[1-\frac{\alpha^{2}}{2 n^{2}}-\frac{\alpha^{4}}{2 n^{4}}\left(\frac{n}{|k|}-\frac{3}{4}\right)\right] .
$$

It is important to note that energy here is defined on an absolute scale. If we ignore the third term of this equation and define it as an approximation, Equation (4) can be written as follows. 


$$
\begin{aligned}
E & =m_{\mathrm{e}} c^{2}-\frac{1}{2}\left(\frac{1}{4 \pi \varepsilon_{0}}\right)^{2} \frac{m_{\mathrm{e}} e^{4}}{\hbar^{2}} \frac{1}{n^{2}} \\
& =m_{\mathrm{e}} c^{2}+E_{n} .
\end{aligned}
$$

$E$ of Equation (5) defines an absolute quantity, which includes the electron's rest mass energy.

Whereas $E_{n}$ in Equation (3) expresses the reduction in rest mass energy of the electron, $E$ in Equation (5) expresses the remaining amount of rest mass energy of the electron.

From Equations (1) and (5) it is evident that, if a stationary electron begins to move in free space, or is incorporated into an atom, then the energy which serves as the departure point is the rest mass energy.

Incidentally, when $n /|k|=1$ in Equation (4), Equation (4) becomes as follows.

$$
E_{n}=m_{\mathrm{e}} c^{2}\left(1-\frac{\alpha^{2}}{2 n^{2}}-\frac{\alpha^{4}}{8 n^{4}}\right) .
$$

Next, let us find the energy of the hydrogen atom from Equation (2). First, Equation (2) can be rewritten as follows:

$$
E_{\mathrm{re}, n}=m_{\mathrm{e}} c^{2}\left(1-\frac{\boldsymbol{p}_{n}^{2}}{m_{\mathrm{e}}^{2} c^{2}}\right)^{1 / 2} .
$$

Also, Equation (3) can be expressed with the following equation:

$$
E_{n}=-\frac{\alpha^{2} m_{\mathrm{e}} c^{2}}{2 n^{2}}
$$

Here, $\alpha$ is the following fine structure constant.

$$
\alpha=\frac{e^{2}}{4 \pi \varepsilon_{0} \hbar c}=7.2973525698 \times 10^{-3} .
$$

Based on this Equation (2) can be written as follows:

$$
\left(m_{\mathrm{e}} c^{2}\right)^{2}\left(1-\frac{\alpha^{2}}{2 n^{2}}\right)^{2}+\boldsymbol{p}_{n}^{2} c^{2}=\left(m_{\mathrm{e}} c^{2}\right)^{2} .
$$

Expanding this equation, the following equation can be obtained:

$$
\boldsymbol{p}_{n}^{2}=\left(m_{\mathrm{e}} c\right)^{2}\left(\frac{\alpha^{2}}{n^{2}}-\frac{\alpha^{4}}{4 n^{4}}\right) .
$$

Incidentally, since $\alpha^{4}=2.8357 \times 10^{-9}$, if this is approximated here as $\alpha^{4} / 4 n^{4} \approx 0$, then Equation (11) can be written as follows:

$$
p_{n} \approx \frac{\alpha m_{\mathrm{e}} c}{n} .
$$

Next, Equation (7) can be written as follows using the relationship in Equation (12):

$$
E_{\mathrm{re}, n}=m_{\mathrm{e}} c^{2}\left(1-\frac{\alpha^{2}}{n^{2}}\right)^{1 / 2} .
$$

The following equation can be obtained by using the expansion of the binomial theorem: 


$$
E_{\mathrm{re}, n}=m_{\mathrm{e}} c^{2}\left(1-\frac{\alpha^{2}}{2 n^{2}}-\frac{\alpha^{4}}{8 n^{4}}+\cdots\right) .
$$

Multiple calculations are needed to derive the energy level (6) from the Dirac equation, but Equation (14) can be easily obtained by starting from Equation (2). (The 4th term exists even in the Dirac equation, but it has simply been omitted.) In the following section, an equation thought to be equivalent to the Dirac equation is derived from Equation (2).

\section{Derivation of an Equation Describing the States of the Electron in the Hydrogen Atom}

Here, the author will review the method of deriving the Dirac relativistic wave equation, for use as reference when deriving the unknown equation.

First, the following quantization is performed for $E$ and $\boldsymbol{p}$ in Equation (1):

$$
E \rightarrow i \hbar \frac{\partial}{\partial t}, \quad \boldsymbol{p} \rightarrow-i \hbar \nabla
$$

Then the following Klein-Gordon equation can be derived:

$$
-\hbar^{2} \frac{\partial^{2}}{\partial t^{2}} \psi=-\hbar^{2} c^{2} \nabla^{2} \psi+m_{\mathrm{e}}^{2} c^{4} \psi
$$

This equation described the wave function in relativistic terms, but the interpretation was inconsistent with the interpretation according to the more commonly used Schrödinger equation.

Equation (16) is a first-order differential equation with regard to space, but it is second-order with regard to time. However, since the theory of relativity handles time and space on equal terms, Dirac believed that it was necessary to derive a first-order differential equation with regard to time. Then he made an assumption, taking the following differential equation as a starting point (Dirac, 1978).

$$
i \hbar \frac{\partial}{\partial t} \psi(\mathbf{r}, t)=H \psi(\mathbf{r}, t)
$$

Dirac also assumed a Hamiltonian $H$ like the following:

$$
H=-i \hbar c \boldsymbol{\alpha} \cdot \nabla+\beta m_{\mathrm{e}} c^{2} .
$$

In the above, $\alpha$ is a 3 -dimensional vector, and $\beta$ is a 1 -dimensional constant.

Here, if Equation (18) is differentiated one more time, and it is assumed that this satisfies Equation (16), then the following relationship is obtained:

$$
\left(-i \hbar c \boldsymbol{\alpha} \cdot \nabla+\beta m_{\mathrm{e}} c^{2}\right)^{2}=-\hbar^{2} c^{2} \nabla^{2}+m_{\mathrm{e}}^{2} c^{4} .
$$

If this equation, is written using the components of $\alpha$, then the result is as follows:

$$
-\hbar^{2} c^{2} \sum_{j, k=1}^{3} \alpha_{j} \alpha_{k} \nabla_{j} \nabla_{k}-i m_{\mathrm{e}} c^{3} \hbar \sum_{j=1}^{3}\left(\alpha_{j} \beta+\beta \alpha_{j}\right) \nabla_{j}+\beta^{2} m_{\mathrm{e}}^{2} c^{4}=-\hbar^{2} c^{2} \sum_{j=1}^{3} \nabla_{j}^{2}+m_{\mathrm{e}}^{2} c^{4} .
$$

This shows that the constants $\alpha$ and $\beta$ introduced here must satisfy relationships like the following:

$$
\beta^{2}=1, \quad \alpha_{j} \beta+\beta \alpha_{j}=0, \quad \alpha_{j}^{2}=1, \quad \alpha_{j} \alpha_{k}+\alpha_{k} \alpha_{j}=0, \quad j, k=1,2,3(j \neq k) .
$$

It is known that the simplest matrix which satisfies these relationships is a matrix with four rows and four columns. Here, the following Pauli spin matrices and the unit matrix are used:

$$
\sigma_{1}=\left(\begin{array}{ll}
0 & 1 \\
1 & 0
\end{array}\right), \quad \sigma_{2}=\left(\begin{array}{cc}
0 & -i \\
i & 0
\end{array}\right), \quad \sigma_{3}=\left(\begin{array}{cc}
1 & 0 \\
0 & -1
\end{array}\right), \quad I=\left(\begin{array}{ll}
1 & 0 \\
0 & 1
\end{array}\right) .
$$

When this is done, the following form can be used as a matrix with four rows and four columns satisfying the condition in (21): 


$$
\alpha_{j}=\left(\begin{array}{ll}
0 & \sigma_{j} \\
\sigma_{j} & 0
\end{array}\right), \quad \beta=\left(\begin{array}{ll}
I & 0 \\
0 & -I
\end{array}\right) .
$$

The Dirac equation can be written as follows using this matrix.

$$
i \hbar \frac{\partial}{\partial t} \psi=\left(-i \hbar c \alpha_{j} \nabla_{j}+\beta m_{\mathrm{e}} c^{2}\right) \psi
$$

Here, the wave function $\psi$ is indicated by a matrix with four rows and one column.

An equation corresponding to the relationship between Equation (1) and Equation (24) will now be derived by referring to the above derivation process (Suto, 2011).

Now, when we perform quantization on Equation (2), we obtain the following:

$$
-\hbar^{2} \frac{\partial^{2}}{\partial t^{2}} \psi=\hbar^{2} c^{2} \nabla^{2} \psi+m_{\mathrm{e}}^{2} c^{4} \psi
$$

This equation corresponds to the Klein-Gordon equation (16) obtained by quantizing Equation (1).

Next, the Hamiltonian describing the state of the electron in the hydrogen atom is assumed to be as follows:

$$
H=-i \hbar c \boldsymbol{\alpha}^{\prime} \cdot \nabla+\beta^{\prime} m_{\mathrm{e}} c^{2} .
$$

Here, if Equation (26) is differentiated one more time, and it is assumed that this satisfies Equation (25), then the following relationship is obtained:

$$
\left(-i \hbar c \boldsymbol{\alpha}^{\prime} \cdot \nabla+\beta^{\prime} m_{\mathrm{e}} c^{2}\right)^{2}=\hbar^{2} c^{2} \nabla^{2}+m_{\mathrm{e}}^{2} c^{4} .
$$

If this equation is written using the components of $\alpha^{\prime}$, the result is as follows:

$$
-\hbar^{2} c^{2} \sum_{j, k=1}^{3} \alpha_{j}^{\prime} \alpha_{k}^{\prime} \nabla_{j} \nabla_{k}-i m_{\mathrm{e}} c^{3} \hbar \sum_{j=1}^{3}\left(\alpha_{j}^{\prime} \beta^{\prime}+\beta^{\prime} \alpha_{j}^{\prime}\right) \nabla_{j}+\beta^{\prime 2} m_{\mathrm{e}}^{2} c^{4}=\hbar^{2} c^{2} \sum_{j=1}^{3} \nabla_{j}^{2}+m_{\mathrm{e}}^{2} c^{4} .
$$

Therefore the constants $\alpha_{j}^{\prime}$ and $\beta^{\prime}$ introduced here must satisfy the following sort of relation:

$$
\beta^{\prime 2}=1, \quad \alpha_{j}^{\prime} \beta^{\prime}+\beta^{\prime} \alpha_{j}^{\prime}=0, \quad \alpha_{j}^{\prime 2}=-1, \quad \alpha_{j}^{\prime} \alpha_{k}^{\prime}+\alpha_{k}^{\prime} \alpha_{j}^{\prime}=0, \quad j, k=1,2,3(j \neq k) .
$$

The following form can be predicted for a matrix with four rows and four columns satisfying these relationships:

$$
\alpha_{j}^{\prime}=i \alpha_{j}=\left(\begin{array}{ll}
0 & i \sigma_{j} \\
i \sigma_{j} & 0
\end{array}\right), \quad \beta^{\prime}=\beta=\left(\begin{array}{ll}
I & 0 \\
0 & -I
\end{array}\right) .
$$

Using this matrix, the equation to be derived can be written as follows:

$$
\begin{aligned}
i \hbar \frac{\partial}{\partial t} \psi & =\left(-i \hbar c \alpha_{j}^{\prime} \nabla_{j}+\beta^{\prime} m_{\mathrm{e}} c^{2}\right) \psi \\
& =\left(\hbar c \alpha_{j} \nabla_{j}+\beta m_{\mathrm{e}} c^{2}\right) \psi .
\end{aligned}
$$

In the era of Dirac, it was thought that Equation (1) could be applied to an electron in free space, and that the following equation incorporating potential energy into Eq. (24) could be applied to an electron in an atom.

$$
i \hbar \frac{\partial}{\partial t} \psi=\left(-i \hbar c \alpha_{j} \nabla_{j}+\beta m_{\mathrm{e}} c^{2}+V\right) \psi
$$

However, if Equation (1) and Equation (32) are compared, the difference between the two forms is too great. To enable application in the atom of Equation (24) which holds in free space, Dirac assumed Equation (32) incorporating potential energy.

If potential energy is incorporated into Equation (1) by applying the same logic, it should be possible to derive an equation applicable to an electron in an atom. Equation (2) was derived based on this sort of logic. 


\section{Theoretical Investigation}

The author had previously believed that, if Equation (31) could be solved, it would be possible to derive an energy level of the hydrogen atom. However, the solution obtained from Equation (32) was the following:

$$
E_{\mathrm{re}, n}= \pm c \sqrt{m_{\mathrm{e}}^{2} c^{2}-p_{n}^{2}}
$$

This was the same result as when the following solution was obtained by solving Equation (27) (Dirac, 1978):

$$
E= \pm c \sqrt{m_{\mathrm{e}}^{2} c^{2}+p^{2}}
$$

Thus the author decided to postpone the solution of this problem to another time. This paper examines what can be predicted from Equation (2) with the levels in classical theory.

First, Equation (2) can be rewritten as follows:

$$
\left[m_{\mathrm{e}} c^{2}+\frac{V(x)}{2}\right]^{2}+p_{n}^{2} c^{2}=\left(m_{\mathrm{e}} c^{2}\right)^{2}
$$

Also, the potential energy in the hydrogen atom can be expressed with the following equation:

$$
V_{n}=-\frac{1}{4 \pi \varepsilon_{0}} \frac{e^{2}}{r_{n}}=-\frac{\alpha \hbar c}{r_{n}}
$$

Now, if this value is substituted into Equation (35), the result is as follows:

$$
\left(m_{\mathrm{e}} c^{2}-\frac{\alpha \hbar c}{2 r_{n}}\right)^{2}+p_{n}^{2} c^{2}=\left(m_{\mathrm{e}} c^{2}\right)^{2}
$$

Expanding and rearranging this equation, it is possible to obtain the following quadratic equation for $r_{n}$ :

$$
p_{n}^{2} r_{n}^{2}-\alpha \hbar m_{\mathrm{e}} c r_{n}+\frac{\alpha^{2} \hbar^{2}}{4}=0 .
$$

If the value of Equation (11) is substituted here for $p_{n}$, the following solution is obtained:

$$
r_{n}=\frac{\alpha \hbar m_{\mathrm{e}} c \pm \sqrt{\alpha^{2} \hbar^{2} m_{\mathrm{e}}^{2} c^{2}-p_{n}^{2} \alpha^{2} \hbar^{2}}}{2 p_{n}^{2}}=\left[r_{\mathrm{e}} \pm r_{\mathrm{e}}\left(1-\frac{\alpha^{2}}{2 n^{2}}\right)\right] \frac{n^{2}}{2 \alpha^{2}}\left(1-\frac{\alpha^{2}}{4 n^{2}}\right)^{-1} .
$$

Here too, let us use both of the two solutions, in the same way that Dirac did not discard the negative solution.

Now, if $r_{n}{ }^{+}$is taken to be the radius when using the positive solution in Equation (39), then $r_{n}{ }^{+}$takes the following value:

$$
r_{n}^{+}=\frac{n^{2}}{\alpha^{2}} r_{\mathrm{e}}=n^{2} a_{\mathrm{B}}
$$

On the other hand, if $r_{n}{ }^{-}$is taken to be the radius when using the negative solution in Equation (39), then $r_{n}{ }^{-}$takes the following value:

$$
r_{n}^{-}=\frac{r_{\mathrm{e}}}{4}\left(1-\frac{\alpha^{2}}{4 n^{2}}\right)^{-1}, \quad \frac{r_{\mathrm{e}}}{4}<r_{n}^{-} .
$$

These values of $r_{n}{ }^{+}$and $r_{n}{ }^{-}$are substituted into the following equation:

$$
E_{n}=-\frac{1}{2} \frac{1}{4 \pi \varepsilon_{0}} \frac{e^{2}}{r_{n}}
$$

If the energies obtained at that time are assumed to be $E_{n}{ }^{+}$and $E_{n}^{-}$, then the following solutions are obtained: 


$$
\begin{gathered}
E_{n}^{+}=-\frac{\alpha^{2} m_{\mathrm{e}} c^{2}}{2 n^{2}} . \\
E_{n}^{-}=-2 m_{\mathrm{e}} c^{2}+\frac{\alpha^{2} m_{\mathrm{e}} c^{2}}{2 n^{2}} .
\end{gathered}
$$

If these energies are described on an absolute scale using $E_{\mathrm{re}, n}$ defined in Equation (2), the results are as follows:

$$
\begin{aligned}
& E_{\mathrm{re}, n}^{+}=m_{\mathrm{e}} c^{2}+E_{n}^{+}=m_{\mathrm{e}} c^{2}-\frac{\alpha^{2} m_{\mathrm{e}} c^{2}}{2 n^{2}} . \\
& E_{\mathrm{re}, n}^{-}=m_{\mathrm{e}} c^{2}+E_{n}^{-}=-m_{\mathrm{e}} c^{2}+\frac{\alpha^{2} m_{\mathrm{e}} c^{2}}{2 n^{2}} .
\end{aligned}
$$

If the above is indicated graphically, the result is as follows (see Figure 1).

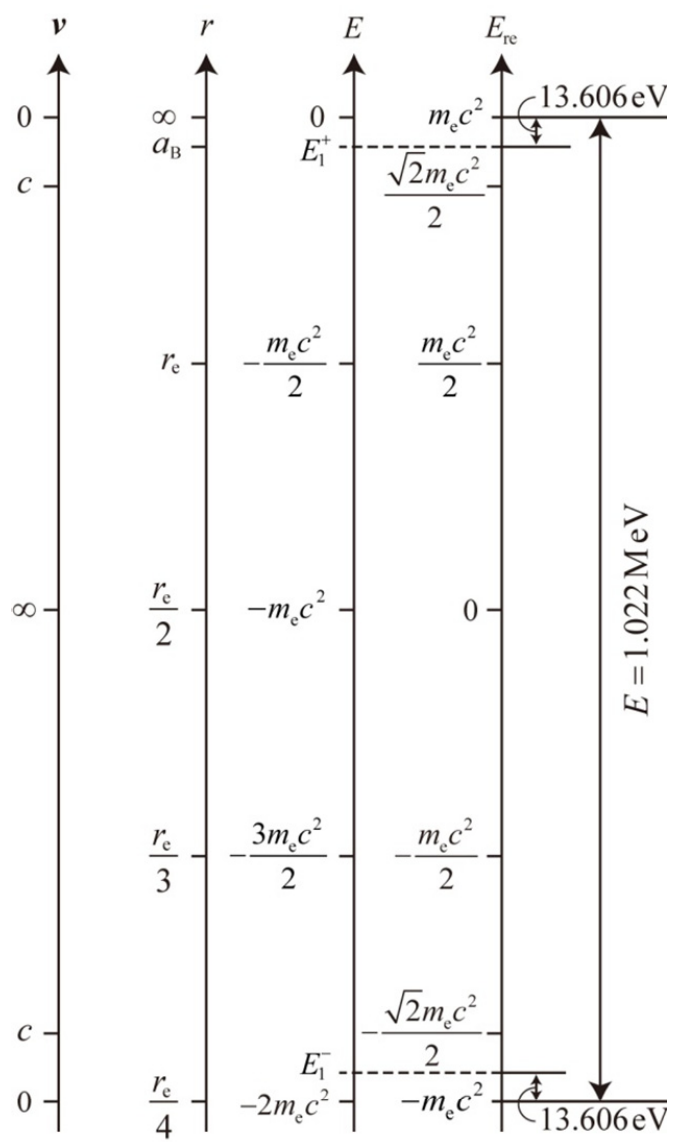

Figure 1 . The energy level of the hydrogen atom predicted by classical quantum theory $E_{n}{ }^{+}$and the new energy level whose existence has been indicated by this paper $E_{n}^{-}$

To make the diagram (Figure 1) easier to see, the positions of $E_{1}^{+}$and $E_{1}^{-}$have been drawn exaggerating the energy width. Also, $r$ and $v$ are physical quantities with meaning within the scope of classical discussions. If the discussion is based on classical considerations, then the velocity of the electron as a particle will exceed the speed of light, and will become infinite at $r=r_{\mathrm{e}} / 2$.

The next step will be to confirm that the electron orbital radius $r$ derived in this paper is a meaningful physical quantity when discussed within the scope of classical quantum theory (i.e., whether $r$ has meaning as an expected value in quantum mechanics).

Incidentally, $E_{\mathrm{re}}=0$ and $E=-m_{\mathrm{e}} c^{2}$ are the same amount of energy, as is evident from Equation (2). Now if Equation (42) is also taken into account, the following equation will hold for an electron with this energy. 


$$
-\frac{1}{2} \frac{1}{4 \pi \varepsilon_{0}} \frac{e^{2}}{r}=-m_{\mathrm{e}} c^{2}
$$

The following value can be obtained from this equation:

$$
r=\frac{1}{2} \frac{1}{4 \pi \varepsilon_{0}} \frac{e^{2}}{m_{\mathrm{e}} c^{2}}=\frac{r_{\mathrm{e}}}{2}
$$

Here, $r_{\mathrm{e}}$ is the classical electron radius.

Furthermore, it is clear from Equations (41) and (44) that the $r$ corresponding to an energy of $E=-2 m_{\mathrm{e}} c^{2}$ is $r_{\mathrm{e}} / 4$. The relationship between $E$ and $r$ is confirmed here because it will be necessary for the discussion in the following section.

\section{Experimental Support}

In this section presents strong evidence providing certainty that electrons exist at the $E_{n}{ }^{-}$energy level.

If it is assumed that the energy of an incident $\gamma$-ray is $1.022 \mathrm{MeV}$ or higher $\left(2 m_{\mathrm{e}} c^{2}\right.$ or higher), i.e., if the energy corresponds to the sum of the mass of the electron and positron, then due to the effects of the Coulomb potential of atomic nucleus, the $\gamma$-ray may suddenly disappear and produce an electron-positron pair (electron-pair creation). According to Dirac's hole theory, if an electron with an energy of $E=-2 m_{\mathrm{e}} c^{2}$ absorbs an energy of $1.022 \mathrm{MeV}$ or higher, the electron will acquire mass and pop out from the vacuum. The hole in the vacuum which remains can be regarded as a positron. However, the fact that electrons and positrons are handled assymetrically is regarded as a difficulty with this theory.

According to the author's ideas, both the electron and positron produced through pair creation in this situation are particles with an energy of $-m_{\mathrm{e}} c^{2}(-0.511 \mathrm{MeV})$ prior to their creation (if the energy of each particle is described using $E_{\mathrm{re}}$, then $E_{\mathrm{re}}=0$ ). Also, the electron and positron pair, which absorbed all of the energy of the $\gamma$-ray, are produced, classically speaking, near $r_{\mathrm{e}} / 2$ from the center of the atomic nucleus (see Figure 2).
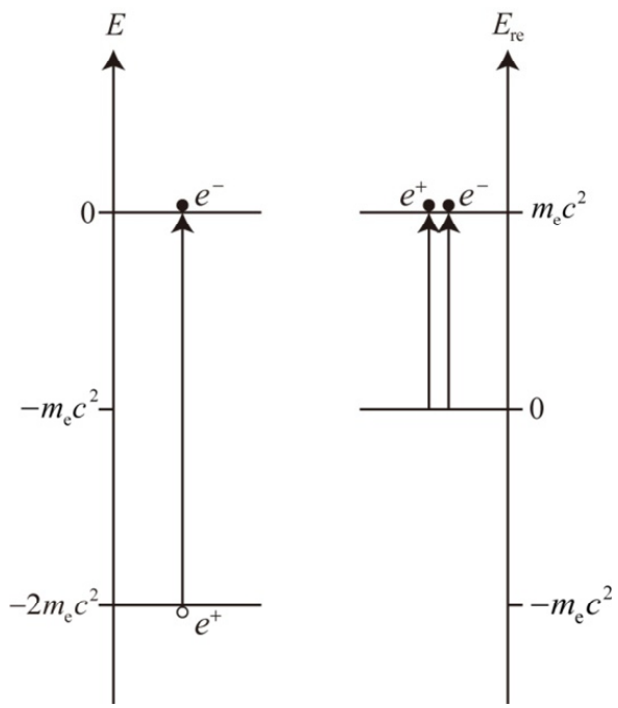

Figure 2. Differences between Dirac's hole theory and the interpration in this paper

Consider the case where a $\gamma$-ray with the energy of 2 electrons $(1.022 \mathrm{MeV})$ is incident on an atomic nucleus (proton). In Dirac's theory, the interpretation is that, when the $\gamma$-ray gives all of its energy to the virtual particles $\left(E=-2 m_{\mathrm{e}} c^{2}\right.$,i.e., $\left.E_{\mathrm{re}}=-m_{\mathrm{e}} c^{2}\right)$ comprising the vacuum around the atomic nucleus, a virtual particle acquires rest mass, and is emitted as an electron into free space, while the hole opened in the vacuum is the anti-particle of the electron. In the author's interpretation, on the other hand, an electron-positron pair is created because a $\gamma$-ray with an energy of $1.022 \mathrm{MeV}$ gives rest mass to a virtual electron-positron pair at the position $r=r_{\mathrm{e}} / 2$. 
In this situation, the energy of the $\gamma$-ray is converted to the rest mass energy of the electron and positron, and the electron and positron are handled on equal terms.

Finally, since the basis of Dirac's hole theory is Equation (1), the energy $E$ of a particle which fills the vacuum is taken to be $E \leq-2 m_{\mathrm{e}} c^{2}$ (i.e., $E_{\mathrm{re}} \leq-m_{\mathrm{e}} c^{2}$ ) (Dirac,1978). In this paper, on the other hand, the discussion is based on Equation (2), and thus no problem arises even if a virtual particle with $E_{\mathrm{re}}=0$ fills the vacuum.

The above reasons explain why a difference arises in the interpretation of electron pair-creation between Dirac and this paper.

Incidentally, if an electron exists at the $E_{n}^{-}$energy level, then the energy $E$ necessary for the electron to be excited and emitted outside the atom is as follows due to Equation (44):

$$
E=2 m_{\mathrm{e}} c^{2}-\frac{\alpha^{2} m_{\mathrm{e}} c^{2}}{2 n^{2}} \approx 2 m_{\mathrm{e}} c^{2}=1.022 \mathrm{MeV} .
$$

Now, consider the case where an incident $\gamma$-ray has the energy corresponding to the mass of 4 electrons (2.044 $\mathrm{MeV})$. This energy is the threshold value for causing triplet production. If this is discussed classically, the $\gamma$-ray can create an electron and positron near $r=r_{\mathrm{e}} / 2$ (see Figure 3).

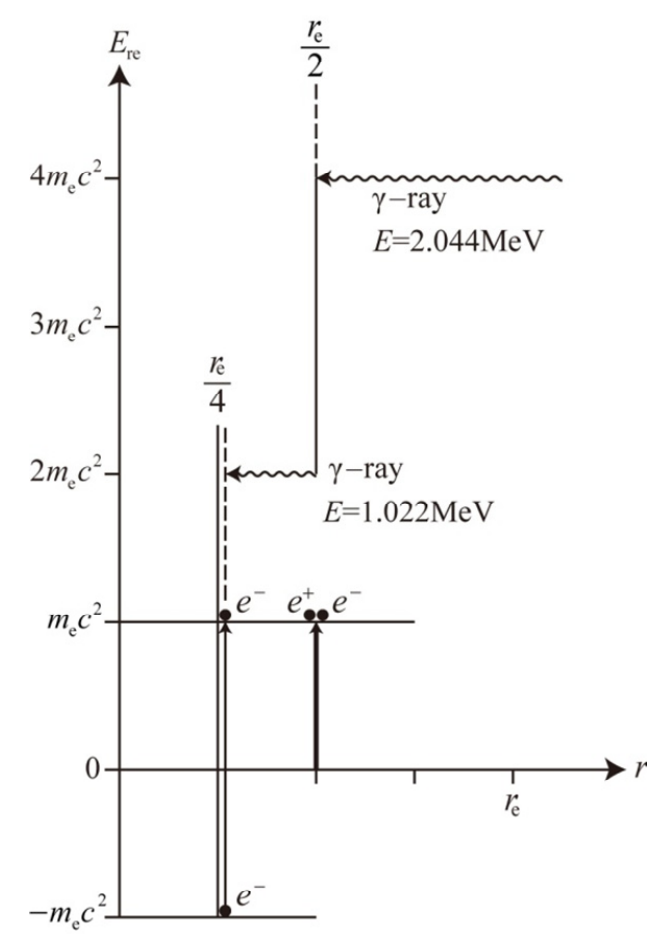

Figure 3. Interpretation of this paper regarding triplet production

Consider the case where a $\gamma$-ray with the energy of 4 electrons $(2.044 \mathrm{MeV})$ is incident on an atomic nucleus (proton). From the perspective of this paper, this $\gamma$-ray will give $1.022 \mathrm{MeV}$ of energy to the virtual particles at $r=r_{\mathrm{e}} / 2$, and an electron-positron pair will be created. After that, if the $\gamma$-ray gives energy to an electron in an electron orbital near the proton, the electron will be excited and appear in free space. As a result, 2 electrons and 1 positron will appear in space. However, according to Dirac's hole theory, when 2 electrons are created, 2 positrons should be created at the same time.

Even if $1.022 \mathrm{MeV}$ of energy is consumed in this pair creation, the $\gamma$-ray still has the energy corresponding to the mass of 2 electrons, i.e., $2 m_{\mathrm{e}} c^{2}(1.022 \mathrm{MeV})$. When this $\gamma$-ray approaches closer to the atomic nuclear (proton), and the electron in the orbital around the proton absorbs this energy, the electron will either be excited to an outer shell orbital, or be emitted to the outside of the atom.

It is generally assumed that in triplet production, in which 2 electrons and 1 positron are created, electron-pair creation occurs not near the atomic nucleus, but near the electron in the outer shell orbital. However, the author 
believes that, if the law of conservation of energy is taken into account, it is more natural to conclude that the electron which recoils is in an extremely low energy state.

If an electron can exist in a state with energy $E_{n}^{-}$, then naturally it can be predicted that other atoms also have similar energy levels. It can also be predicted that these atoms, and molecules produced from these atoms, are a strong candidate for dark matter.

\section{Conclusion}

The author has previously derived relationship (2) relating energy and momentum, which holds in the hydrogen atom, based on Einstein's relationship (1). The energy range where this Equation (2) is applicable is thought simply to be the following range.

$$
-m_{\mathrm{e}} c^{2} \leq E_{\mathrm{re}} \leq m_{\mathrm{e}} c^{2}
$$

Following Dirac, this paper used a negative energy solution which can be derived from Equations (41) and (42). As a result, it was possible to predict that the hydrogen atom has an extremely low energy level.

The known energy level $E_{n}{ }^{+}$and the unknown energy level $E_{n}{ }^{-}$are given by the following equations.

$$
\begin{gathered}
E_{n}^{+}=-\frac{\alpha^{2} m_{\mathrm{e}} c^{2}}{2 n^{2}}, \quad n=1,2, \cdots \\
E_{n}^{-}=-2 m_{\mathrm{e}} c^{2}+\frac{\alpha^{2} m_{\mathrm{e}} c^{2}}{2 n^{2}}, \quad n=1,2, \cdots .
\end{gathered}
$$

If these equations are written from an absolute standpoint using the energy $E_{\mathrm{re}, n}$ the results are as follows:

$$
\begin{gathered}
E_{\mathrm{re}, n}^{+}=m_{\mathrm{e}} c^{2}\left(1-\frac{\alpha^{2}}{2 n^{2}}\right), \quad n=1,2, \cdots \\
E_{\mathrm{re}, n}^{-}=-m_{\mathrm{e}} c^{2}\left(1-\frac{\alpha^{2}}{2 n^{2}}\right), \quad n=1,2, \cdots .
\end{gathered}
$$

However, Equations (51) to (54) are approximations, as is also clear from the fact that Equation (3) is an approximation of Equation (6).

Recently, the author has presented the following equation, which is more precise than the previous equation, as the energy levels of the hydrogen atom including only the principal quantum number $n$ (Suto, 2014).

$$
E_{n}=m_{\mathrm{e}} c^{2}\left[\left(\frac{n^{2}}{n^{2}+\alpha^{2}}\right)^{1 / 2}-1\right], \quad n=1,2, \cdots .
$$

This is an abrupt note, but if that result is taken into consideration, it can be concluded that the following equation has higher precision than Equations (53) and (54).

$$
\begin{aligned}
& E_{\mathrm{re}, n}^{+}=m_{\mathrm{e}} c^{2}\left(\frac{n^{2}}{n^{2}+\alpha^{2}}\right)^{1 / 2}, \quad n=1,2, \cdots \\
& E_{\mathrm{re}, n}^{-}=-m_{\mathrm{e}} c^{2}\left(\frac{n^{2}}{n^{2}+\alpha^{2}}\right)^{1 / 2}, \quad n=1,2, \cdots
\end{aligned}
$$

\section{Acknowledgements}

I would like to express my thanks to the staff at ACN Translation Services for their translation assistance. Also, I wish to express my gratitude to Mr. Hiroshi Shimada for drawing figures.

\section{References}

Dirac, P. A. M. (1978). Directions in Physics (p. 11, 12, 14). New York: Wiley. 
Schiff, L. I. (1968). Quantum Mechanics (p. 472). New York: McGraw-Hill.

Suto K. (2011). Alternative formulation of relativistic quantum mechanics, Physics Essays, 24(4), 598-602. http://dx.doi.org/10.4006/1..3659280

Suto K. (2011). An energy-momentum relationship for a bound electron inside a hydrogen atom. Physics Essays, 24(2), 301-307. http://dx.doi.org/10.4006/1.3583810

Suto K. (2014). n=0 energy level present in the hydrogen atom. Applied Physics Research, 6(5), 109-115. http://dx.doi.org/10.5539/apr.v6n5p109

\section{Copyrights}

Copyright for this article is retained by the author(s), with first publication rights granted to the journal.

This is an open-access article distributed under the terms and conditions of the Creative Commons Attribution license (http://creativecommons.org/licenses/by/3.0/). 